\title{
INVESTIGATION OF THE UNDERGROUND BUILDING HERITAGE AND THE MECHANISM OF WATER FLOWING IN QANĀTS IN PALERMO THROUGH INNOVATIVE SURVEYING TECHNIQUES
}

\author{
R. Corrao ${ }^{1}$, F. Di Paola ${ }^{1 *}$, D. Termini ${ }^{2}$, C. Vinci $^{1}$ \\ ${ }^{1}$ DARCH, Department of Architecture, 90128 Palermo, Italy - (rossella.corrao, francesco.dipaola, calogero.vinci)@unipa.it \\ ${ }^{2}$ DING, Department Engineering, 90128 Palermo, Italy - donatella.termini@unipa.it
}

KEY WORDS: Underground building heritage, qanāt, SLAM, wearable mobile laser system, 3D digitalization, visualization, cultural heritage.

\begin{abstract}
:
The valorisation, protection and preservation policies for the underground building heritage are often difficult to implement due to an inadequate knowledge of hypogeal constructions. The complex and widespread underground structures of the vast "Cavo" Heritage ("horizontal wells", "shelf wells" or "well tunnels"), so called qanäts, hidden underground and built over the centuries in Palermo, representing an evocative testimony to the history of water culture in the ancient city. Through the historical and constructive analyses and the implementation and development of measurement and 3D representation and visualization, first actions have been carried out. The paper will present the first results of the restoration project and the path of re-introduction in the fruition network of the qanāt "Gesuitico alto", developed also in the field of "iHeritage. Mediterranean Platform for UNESCO Cultural Heritage" project, financed by ENI CBC MED Programme 2014-2020. The paper presents an experimentation of a procedural workflow of data acquisition, analysis and subsequent 3D virtual navigation of hypogeal environments. The methodology used is the SLAM with a last generation WMLS. The platform of virtual reality visualization, within UnReal Engine, allows the user to immerse and navigate in the anthropic environment by engaging it with a set of infographics that highlight the virtual visit.
\end{abstract}

\section{INTRODUCTION}

The valorization, protection and preservation policies for the underground building heritage are often difficult to implement due to an inadequate knowledge of hypogeal constructions. The fragmented and incomplete documentation is, often, due to the lack of systematization of multidisciplinary studies carried out in a unique typological context and related to only apparently episodic discoveries; to the difficulties to access to underground architectures and their complexity; to problems related to safety that require careful exploration programs. The indications formulated and codified in the Athens Charter and, more recently, in the Krakow Charter, define the guidelines for the conservation and enhancement of cultural heritage, emphasizing the strategic importance of multidisciplinary scientific approaches to the management of interventions in cultural heritage sites (De Naeyer, 2000).

In different urban contexts like in Palermo, indeed, the presence of numerous underground cavities of anthropogenic origin that remain unknown could determine formation of chasms, collapses of streets or buildings above them and consequent disasters and/or inundation phenomena with possible loss of human lives. In particular, the system of groundwater connected conveyance tunnels are of particular interest in Palermo. A detailed survey of the underground systems is extremely important to properly know underground building heritage before eventual disaster can happen. However, the investigation of this underground aqueduct system is, therefore, of considerable complexity since it represents a unicum in terms of typology and construction.

A renewed interest emerged from the studies carried out in the last years. Through the historical and constructive analyses and the implementation and development of measurement and 3D representation and visualization, first actions have been carried out for revaluating the hypogeal building heritage of Palermo, intended as a resource that can be exploited in a perspective of conservation and sustainable fruition.

The complex and widespread underground structures of the vast "Cavo" Heritage ("horizontal wells", "shelf wells" or "well tunnels"), so called qanāts, hidden underground and built over the centuries, representing an evocative testimony to the history of water culture in the ancient city of Palermo (Todaro, 1988; Vinci, 2018). The system of connected qanāts constituted an underground aqueduct able to transport water from an underground natural aquifer to the surface. It identified an original hydraulic system, very similar to the qanāt of the Middle East culture, allowing over the centuries the water supply to the city.

Moreover, the network of qanāts represented the invisible part of a more complex combined system including the so called "Scirocco rooms", used in the villas built by the Palermitan aristocracy between XVI and XVIII centuries in the surrounding area of historical city center (the so called "Conca d'Oro"). Today, this system results partially destroyed because of the construction of the new buildings of modern city that often did not properly considering its presence underground; for these

\footnotetext{
Corresponding author
} 
reasons its surviving traces are very vulnerable and they need to be preserved and valorized.

Under the umbrella of "iHERITAGE. Mediterranean Platform for UNESCO Cultural Heritage" project, financed by ENI CBC MED Program 2014-2020, a multidisciplinary investigation started with the aim to re-discovery and systematize all existing fragments, visible and "invisible", epigean and hypogeal, of the historical system of water management of ancient Palermo. With the logistical and scientific support of "CAI Palermo" speleological group, which for years has been collaborating with the Superintendence of cultural and environmental heritage for the exploration of qanāt, the iHeritage research group is working for the development of an innovative tools that will allow the virtual fruition of qanāt also to people with disabilities that usually cannot have access to qanät.

\subsection{Case Study: the qanāt "Gesuitico alto"}

The network of qanāts in Palermo develops in three different levels, both for the underground bottom depth and for the typology and construction techniques. These aspects, probably, depends on the progressive lowering of the water level and thus to the necessity to reach deeper underground depths to intercept the water. When the lowering of the water level was very rapid (e.g. earthquake in 1693), it was preferred to directly dig a new tunnel at a lower level, also with different construction techniques. This paper presents the preliminary results of the restoration project and the path of re-introduction in the fruition network of "Gesuitico alto" qanāt, an intricate underground architecture made of tunnels simply carved into the rock and/or defined with squared ashlars.

As often happens, in hypogeal buildings, the most superficial level (level 1) of tunnels is the oldest. The unaltered segment of the latter develops to the north for a length of about $155 \mathrm{~m}$, to the south for $77 \mathrm{~m}$, at a depth of about $6 \mathrm{~m}$.

The width, the height and the shape of the section of these tunnels are very variable. An extensive part of the qanāts was almost completely filled with material from the excavation of the lower tunnel. The tunnel level 2, at a depth of 8,50 m, has a length of about $110 \mathrm{~m}$; the section is rectangular, it has variable height and its walls are without lining. Tunnel Level 3 is at a depth of $10 \mathrm{~m}$ and is $502 \mathrm{~m}$ long; the path partially overlaps that of level 1. For this reason, when the distance between the two tunnels became very small, they were joined into a single section with a height varying between 2.50 and $3 \mathrm{~m}$. The tunnel path in levels 1 and 2 is moderately tortuous and deviates in correspondence with more compact rock masses. Level 3 has long straight stretches in which even the most compact rock masses have in many cases been excavated and demolished. This testifies, in addition to the presence of many parts covered with ashlars made of limestone, more advanced excavation and construction techniques.

The lining walls and the covers of the tunnel are made of dry limestone ashlars; this guarantees the stability of the excavation walls in case of not very resistant soils and, at the same time, the drainage of the waters, that occasionally infiltrates from the side walls increasing the flow of the tunnel. The coverage of the channel, in the coated sections, is made as "bocca di forno" type: two ashlars inclined forming a triangular profile. In this way, a pushing structure is created that stabilizes the two side walls of tunnel and it allows to deposit the excavated material on the extrados of the ceiling, avoiding the costly and tiring transport out of the tunnel.

"Corso Gesuitico alto" is, furthermore, a rare testimony of the ancient Sicilian water culture; in fact, it preserves many characteristic elements: the pit for the senia at the end of level 2; the serial aeration pits; a repertoire of catusi (pipes with truncated conical brick segments) of different sizes; barrage systems and numerous historical interventions of restoration and consolidation (figs. 1-2).

\section{MATERIAL AND METHODS}

The survey process cannot disregard the exploration and the non-invasive digital acquisition of underground environments that face the many logistical challenges of capture (presence of humidity, restricted and narrow areas, lack of light, absence of GPS signal, inability to stand or survey, etc.) to detect complex surfaces with high geometric resolution (Zlot et al., 2013; Roncat et al., 2011).

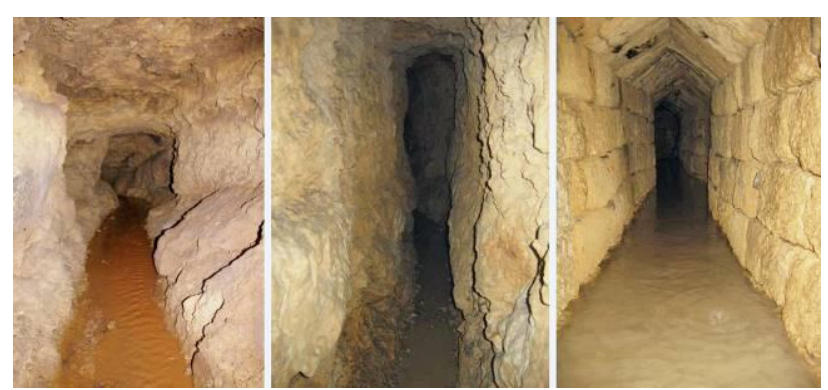

Figure 1. "Corso Gesuitico Alto". From the left, morphology of level 1, level 2 and level 3 sections of tunnels.

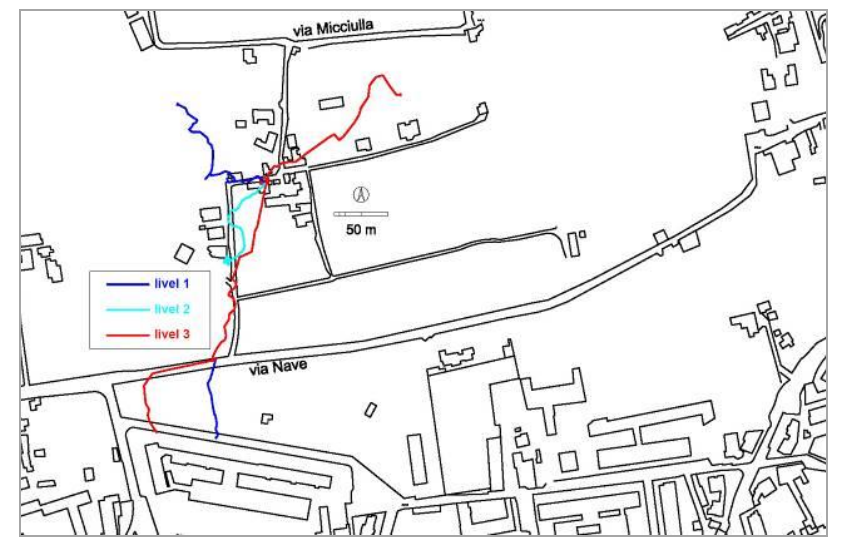

Figure 2. "Corso Gesuitico Alto" in the urban context. (Survey: Gruppo Speleo CAI Palermo. Graphic restitution: INS Salvatore Sammataro, INS Silvia Sammataro - 2014).

The study evaluates the potential of the laser system employed, considering several parameters that influence the final quality of the 3D point cloud (identification of critical areas; prediction for loop closure; the speed of movement; the time taken to obtain the 3D point cloud; the density of the point cloud). 3D realitybased surveying instruments and techniques offers new and effective solutions for the 3D modeling of hypogeal environments (Galeazzi et al., 2014).

In recent years, metric surveying applications with hybrid solutions, such as mobile mapping systems (MMS), have raised great capabilities and possibilities (Nocerino et al., 2017; Vaaja, Virtanen, 2017). In addition to advances in optical sensors, which minimize error propagation, one of the key advances in MMSs is related to spatial reference technology.

In this category of equipment, especially in complex contexts with enclosed and difficult-to-access spaces, which are common morphological features in Cultural Heritage, wearable mobile laser systems (WMLS) present themselves as a potential solution for mapping indoor environments, as a $3 \mathrm{D}$ point cloud 
of the environment can be obtained with an accuracy of $\mathrm{cm}$ precision. However, this accuracy could be strongly affected by trajectory characteristics, such as the speed of movement or the path followed (Lagüela et al., 2018).

In this specific field of investigation, the paper presents an experimentation of a procedural workflow of data acquisition, analysis and subsequent 3D virtual navigation of hypogeal environments in Palermo.

In the following paragraphs will be described the workflow tested, and the considerations that determined the parameter settings and survey strategies. The phases of the acquisition process, 3D data analysis, management and optimization of the digital model set up will be described to obtain a realistic scenario of the hypogeal architectural system for the creation of a virtual tour in gaming modality (Kalay et al., 2007).

Computer Graphics, Web Design and Gaming technologies and with high-performance devices in VR, AR and Mixed Reality allow to generate applications for high-resolution visualization of three-dimensional environments with the ability to navigate, analyse and query virtual models in real time, allowing a navigation experience even for users with physical or perceptual disabilities or special pathologies. Moreover, now more than in the past it is possible to download VR and AR on mobile devices not only for gaming or Cultural Heritage $(\mathrm{CH})$ dissemination, but also for supporting decision-making processes and for planning intervention actions to manage and monitor sites in danger, such as the case study related to Palermo qanāts.

The paper presents a prototype of the virtual reality visualization platform, implemented within the environment UnReal Engine (by Epic Games), allows the user to immerse and navigate in the anthropic environment by engaging it with a set of infographics that highlight the virtual visit by explaining construction techniques and materials (fig. 3).

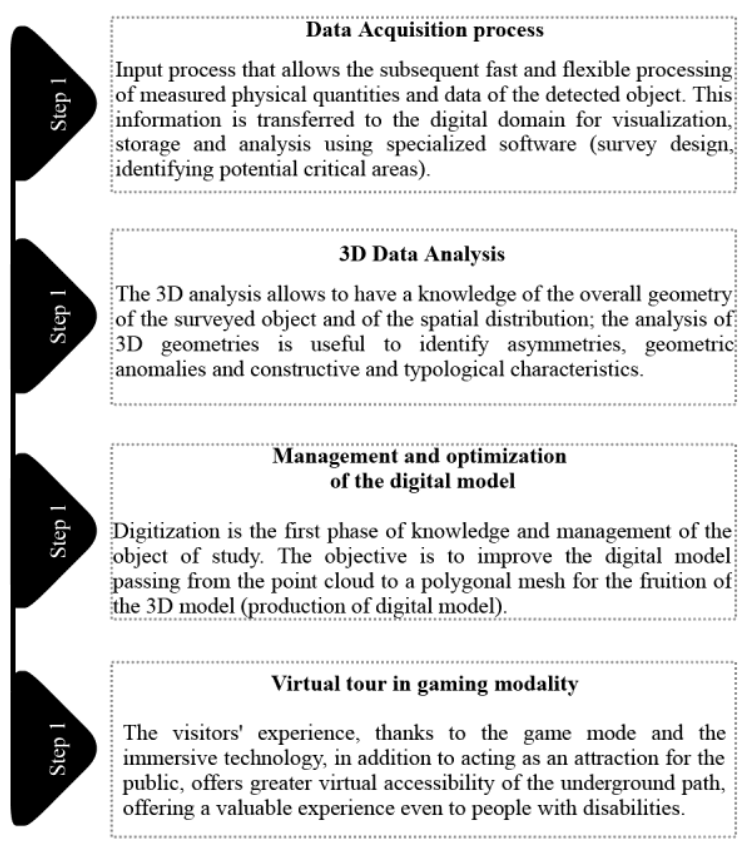

Figure 3. Methodological phases of the procedural pipeline.

2.1 Equipment: The methodology used is the SLAM (Simultaneous Localization and Mapping) with a last generation wearable mobile laser scanner. The WMLS tested in the case study is the Zeb Horizon, commercialized by GeoSLAM (Di Filippo et al., 2018). The instrument provides a rapid means of capturing 3D point cloud data. The data is captured as the user walks through the area of interest. This wearable system combines laser scanning technology (2D time-of-flight laser scanner) and an inertial measurement unit (IMU) in a portable equipment that can be managed by an operator while walking around the site. The sensor captures moving point clouds using Simultaneous Localization and Mapping (SLAM) algorithms (Bosse et al., 2012) without the need for global navigation satellite system (GNSS) support (fig. 4). With a $360^{\circ}$ vertical field of view and $60-80 \mathrm{~m}$ maximum range under indoor conditions (which was reduced to 5-10 m under real-world working circumstances), the operator moved within the hypogeal section capturing about 300.000 points per second. Regarding accuracy, the manufacturer stated it to be $1-3 \mathrm{~cm}$ in relative terms and $1-30 \mathrm{~cm}$ in absolute positioning for a $10 \mathrm{~min}$ scan with a single loop closure. Table 1 shows additional technical specifications.

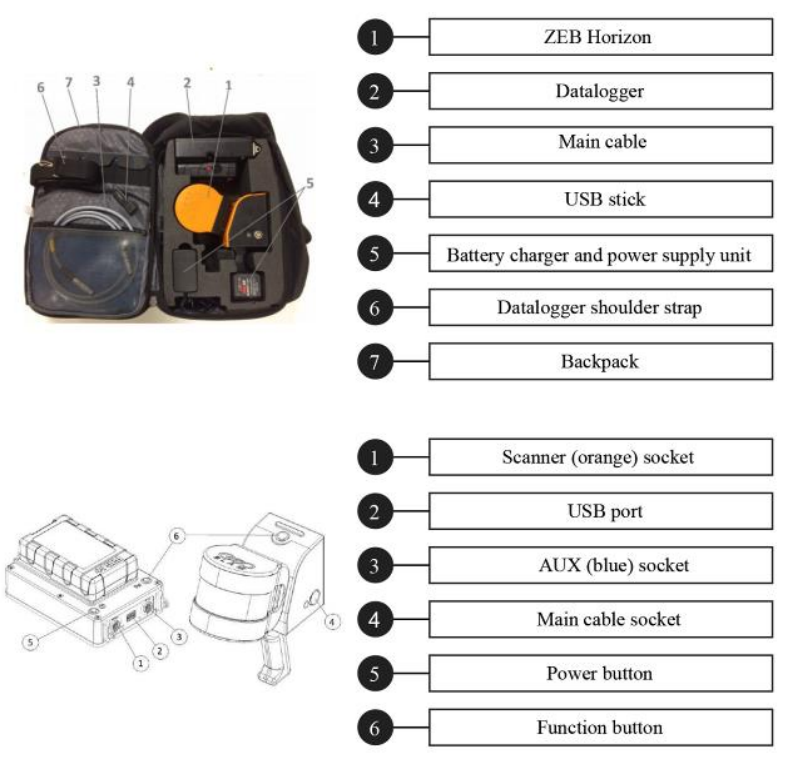

Figure 4. Main components of the wearable mobile laser systems (WMLS) used for data acquisition.

\begin{tabular}{|l|c|}
\hline \multicolumn{2}{|c|}{ Peb Horizon features } \\
\hline Range & Value \\
Protection class & $100 \mathrm{~m}$ \\
Processing & IP54 \\
Data logger carrier & Post \\
Payload & Backpack or shoulder strap \\
Scanner points per second & $3,7 \mathrm{Kg}$ \\
No. of sensors & 300,000 \\
Relative accuracy & 16 \\
Raw data file size & $1-3 \mathrm{~cm}$ \\
Colourised point cloud & $100-200 \mathrm{MB}$ a minute \\
Referenced imagery & $\bullet$ \\
Automated scanner start & $\bullet$ \\
Field of view & $\bullet$ \\
Operating temperature & $360^{\circ} \times 270^{\circ}$ \\
\hline
\end{tabular}

Table 1. Technical specification of the Geo Simultaneous Localization and Mapping (SLAM) ZEB-HORIZON device.

2.2 Acquisition and survey design: The analysis of this complex underground building heritage requires the planning of a specific methodology of investigation.

The first step required a preliminary check carried out with a series of inspections, aimed at identifying potential critical areas 
during the scanning process (obstructed accesses during the path; flooding; danger of collapse; undercuts; prohibitive conditions in relation to the SLAM instrument range). The geometric-formal complexity of the site (rocky and jagged structure with few identifiable landmarks; scarcity of light and operating space; presence of water at different depths) and the type of articulated and practicable path in a single direction of travel have imposed a strategy that would allow to close the loop and, at the same time, to detect the undercut areas. The tunnel has an almost constant width of about $70 \mathrm{~cm}$, while the average height is more than $2 \mathrm{~m}$, but with stretches high only 1 $\mathrm{m}$ and a half, which forces to continue the tour with the bust tilted forward.

For a correct processing, it is, in fact, required that the operator starts and ends the survey in the same position to ensure the closure of the loop. Before starting the acquisition phases, simulations were carried out to evaluate some factors that could have affected the quality of the data flow (speed of movements during the path, variable heights and obstacles that would have slowed down the operator's movements) (fig. 5).
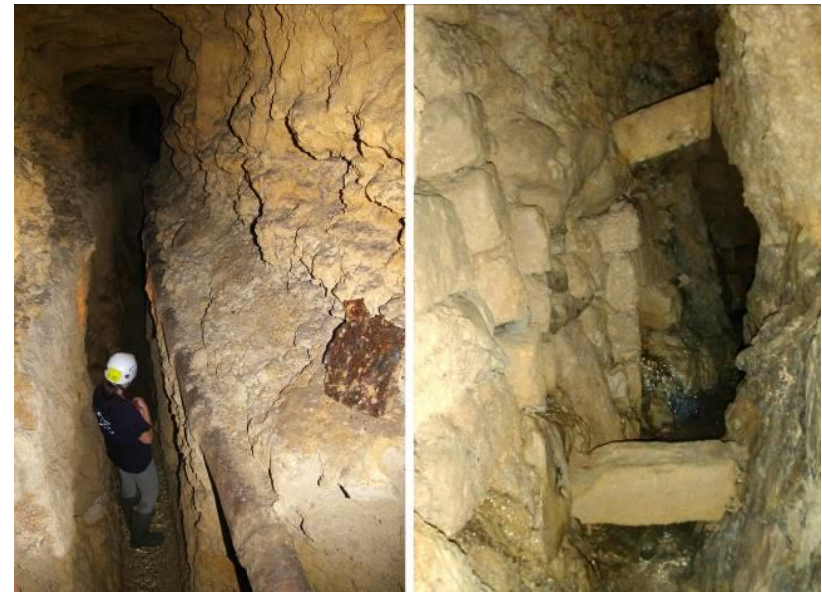

Figure 5. The geometric-formal complexity of the site (Photographic archives CAI Palermo Speleological Group).

During scanning phase, great care was taken with the forward speed to ensure good coverage and high-resolution data. Actually, it is known, that if the forward motion is too fast, there may not be enough repeated feature scans for the SLAM algorithm to process the raw laser data into a point cloud.

In addition, to ensure high data density and not to incur errors while transitioning through survey areas of the qanatt, the scan head was kept stationary for short periods of time to acquire difficult and limited access points.

The SLAM algorithm used to process the raw laser scanning data into a $3 \mathrm{D}$ point cloud relies on there being elements in the scanned environment that are repeatedly scanned as the operator progresses along the path. For an element to be significant, the ratio of its size to its range must be about $1: 10$, e.g. at $5 \mathrm{~m}$ for an element to be significant it must have a size $>0.5 \mathrm{~m}$. In the case study under consideration, excessively narrow spaces or smooth walls are classified as "element-poor" environments. Under these circumstances, there may not be sufficient elements in the direction for the SLAM algorithm to determine forward motion. So, to avoid algorithm processing errors, a route was planned that involved repeated scanning of feature-poor elements.

In addition, the coexistence of confined spaces and complex larger areas required special attention to instrument management in the field. To avoid the recording of large files and reduce possible error propagation, we tried to calibrate, also, the speed of the movements with the duration of the scanning process (recommended at a maximum of 30 minutes) (figs. 6-7). In addition to trajectory calculation, prior planning was done to place the mobile device in an environment that had suitable and safe morphological characteristics.

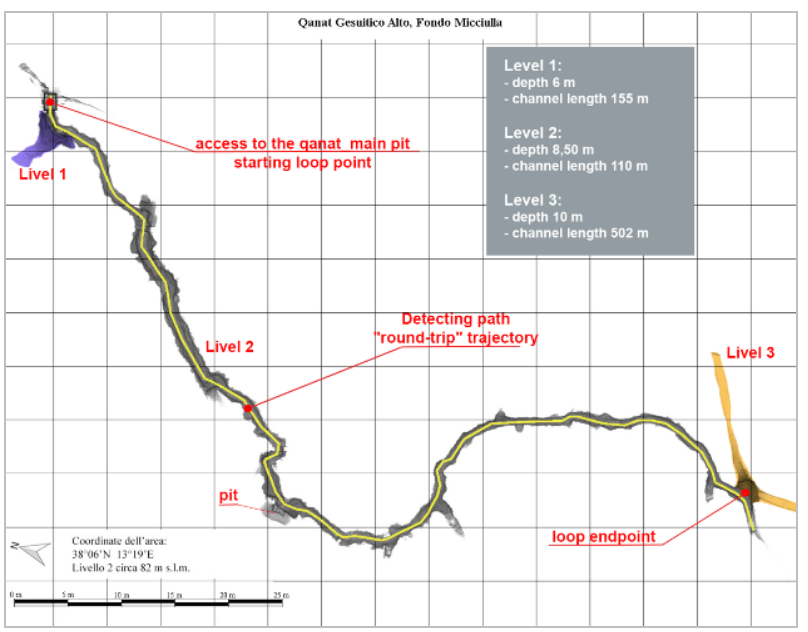

Figure 6. Survey design with the WMLS. Strategy of "roundtrip" trajectory to close the loop and to detect the undercut areas.
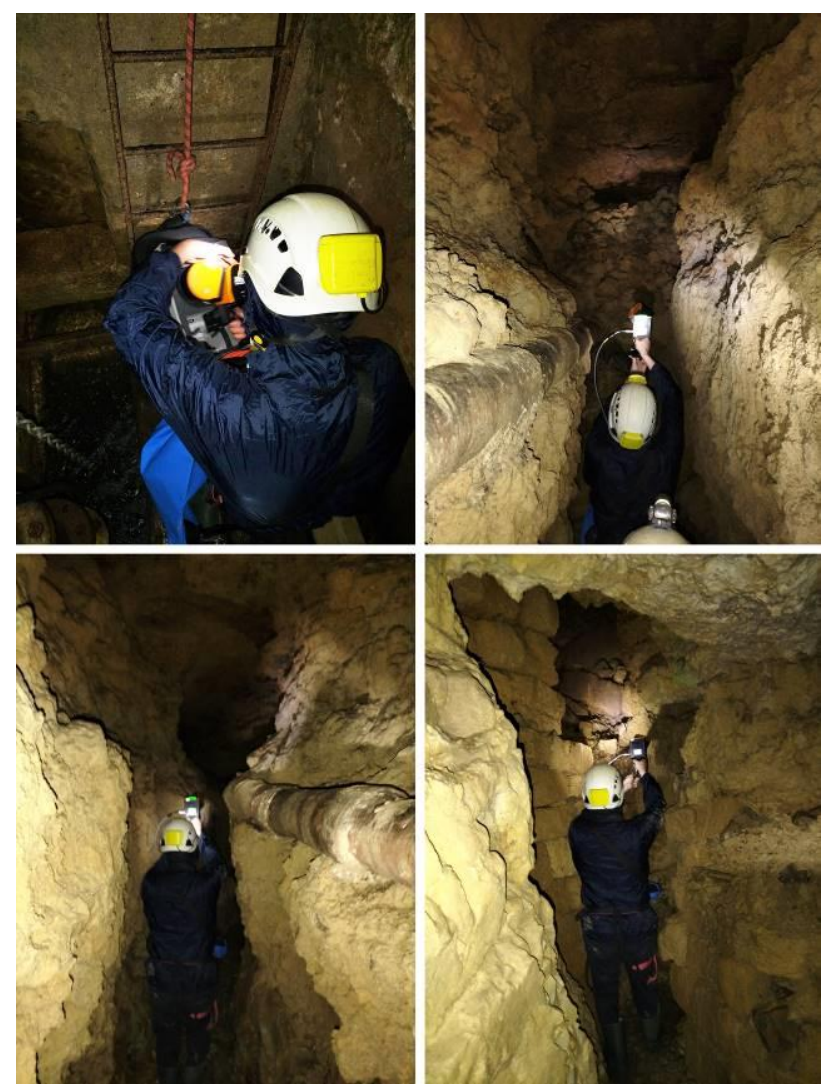

Figure 7. Some phases of the survey in "Gesuitico alto" qanāt. 
2.2.1 Data Processing and Registration: As mentioned above, the instrument captures raw laser distance and inertial data.

This data must be processed using GeoSLAM's SLAM algorithm to convert the raw data into a 3D point cloud. At registration, the data is processed using the processing application GeoSLAM Hub. To understand the performance of the GeoSLAM algorithm, it is appropriate to describe the general characteristics of the algorithm and its operation.

The trajectory reproduced by the software describes the position of the sensor during data acquisition. The 3D SLAM algorithm combines 2D laser scanning data with IMU data, returning accurate 3D point clouds. Data processing requires an incremental procedure (segments are recorded one by one), like the well-known iterative nearest point (ICP) algorithm (Di Filippo et al., 2018).

The registration phase has imported and processed the captured dataset within the proprietary software. This operation allowed the $3 \mathrm{D}$ point cloud to be visualized and post-processed. Given the critical nature of the unique and typologically singular scan area, it was necessary to initiate re-processing options that compensated for drifts and slips caused by inconsistent data due to the presence of scanned areas with few significant features in the $10 \mathrm{~m}$ range.

Some parameters (Local and Global Registration Options) have been set to optimize the final data and compensate for any scanning errors. Two important modified parameters are reported: "Convergence Threshold" and "Voxel Density".

The first one grows the maximum number of iterations per processing step and reduces the convergence threshold during the local registration phase. The second one, however, controls the size of the voxels (cubes) into which the data is divided. Increasing the voxel density decreases the voxel size. Increasing the voxel density will allow more detail to be detected at close range and used during SLAM processing (figs. 8-9).

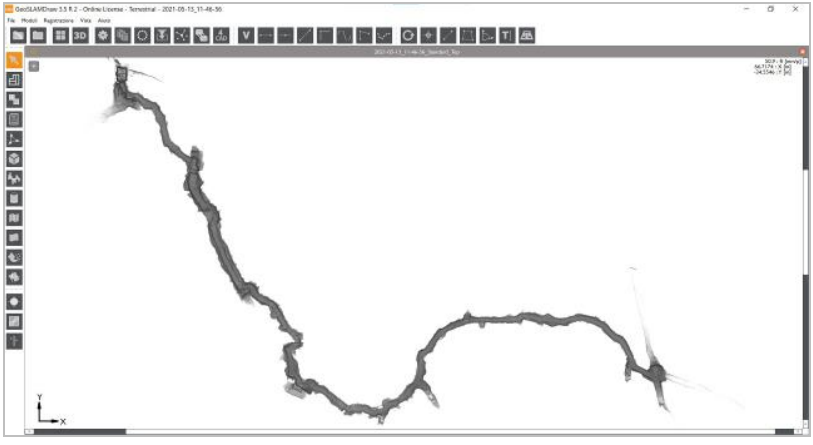

Figure 8. GeoSLAM Draw interface. Top view in orthogonal projection of the point cloud of the "Gesuitico Alto" qanāt.

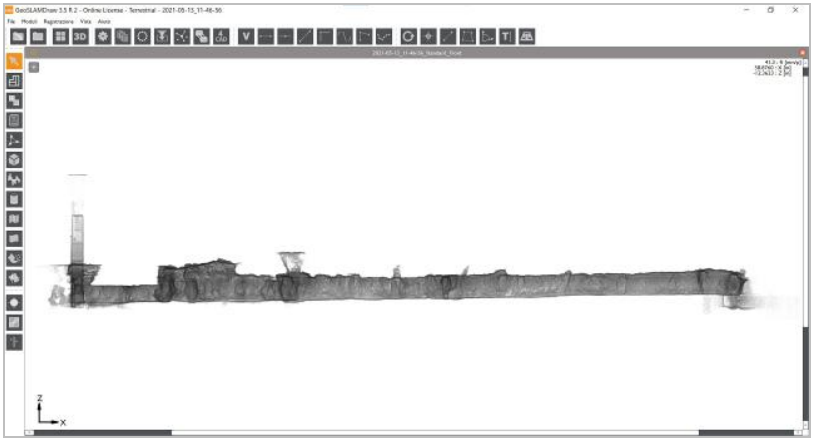

Figure 9. GeoSLAM Draw interface. Side section view in orthogonal projection of the point cloud of "Gesuitico Alto" qanāt.
Increasing these parameters solved some processing errors, but at the expense of processing time.

The GeoSLAM Draw solution for efficient processing of point clouds to create detailed 2D plans and raster views was used to visualize the first results (at depths of field different from the section plane). This initial graphical setup enabled practical functions for measuring and querying the hypogeal system.

2.2.2 Post-Processing: After processing the dataset in GeoSLAM Hub, the next step of the elaboration process was to export the point cloud data in LAS format to be processed in other point cloud processing software. It is important to specify that the format chosen for the export does not support the calculation of the points normals and, therefore, this operation, that is fundamental for the creation of the final model mesh, was carried out later (fig. 10).

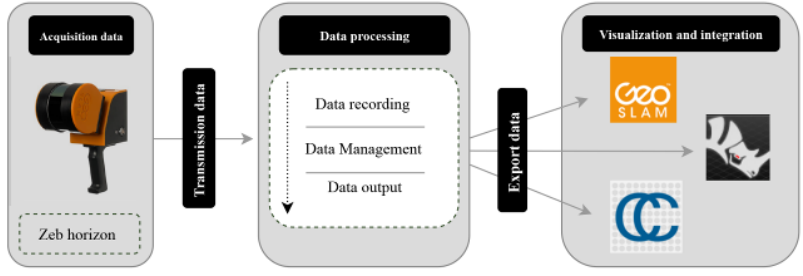

Figure 10. Workflow processing and data integration.

The next step after the first data recording was to process the point cloud in the well-known point cloud processing software, CloudCompare (2.12 alpha). The software was mainly used to optimize the cloud, applying the tools (segmentation, distance computation, spatial operators, colours, normal compute, scalar fields) and plugins, managing the classic octree structure of the program. The main objective was to extrapolate vector profiles in cross-sections orthogonal to the trajectory and longitudinal (fig. 11).

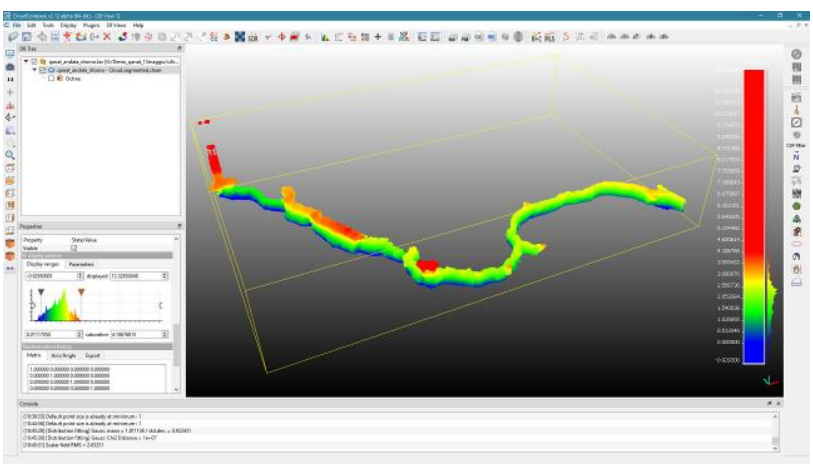

Figure 11. Scalar Fields gradient output and histogram dialog.

To manage the amount of data more efficiently, it was appropriate to segment the cloud into six sections, determined in relation to the morphological characteristics of the entire path. To filter the data, some specific algorithms were used, following a methodological procedure with values and processing criteria for all the portions identified with the aim of eliminating duplicate points and "outliers" by reducing redundant points, while maintaining the cloud with a high information density (the procedures adopted allowed to reduce the cloud from $123,790,232$ points to $72,680,052$ points). In specific, two commands were used, respectively, for the above operations: "subsample a point cloud" and the "SOR filter", in the section tools clean. 
Table 2 shows the values applied for each of the sections. It should be noted that it was not necessary to use the Noise Filter algorithm, capable of reducing noise, since the raw cloud produced by the SLAM methodology was well structured.

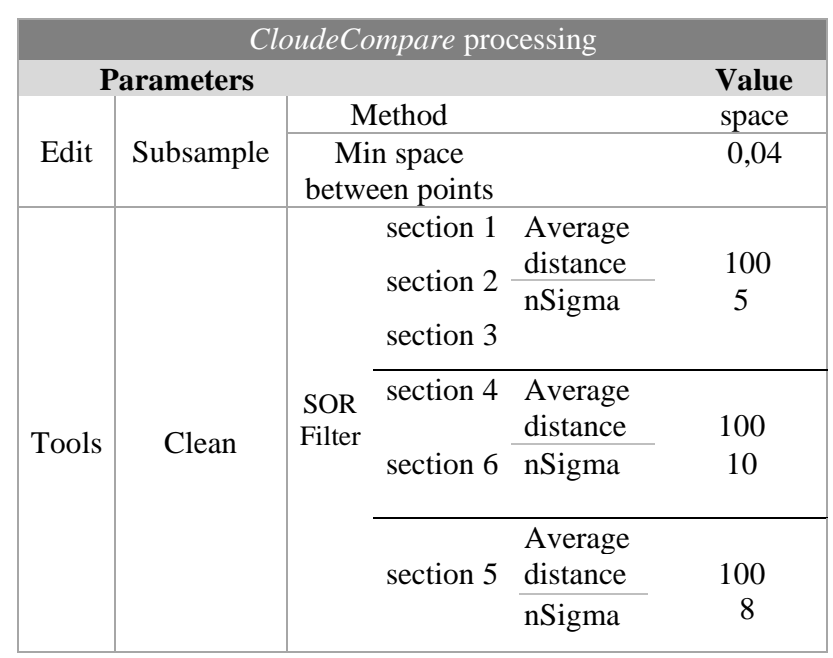

Table 2. Point cloud processing in CloudCompare. Segmentation, applied tools and set parameters.

2.2.3 Plan and Section Restitution: Having still maintained a high density for the reference cloud, longitudinal profiles and a plan profile were extracted. Here again, as with all other procedures, they were performed on a section-by-section basis. Instead to define multiple orthogonal section profiles, it would have been more convenient to define a single path and automatically generate sections orthogonal to the trajectory, travelled by the operator during the scan, at regular intervals (Tools -> Segmentation -> Extract sections).

To maintain more control, it was preferred to use the "cross section" command which segments the selected clouds into smaller parts separated by an assigned distance of $1 \mathrm{~m}$ (in total 103 profiles were extracted). It was not possible to establish this distance with the first procedure mentioned, that gave only the option to determine the number of sections to be made. Our interest was to get more sections at the same time, so we operated through the command "export multiples slices". Obtained file was finally processed in Rhinoceros to obtain the plan and the cross sections of the of the underground building (figs. 12-13).

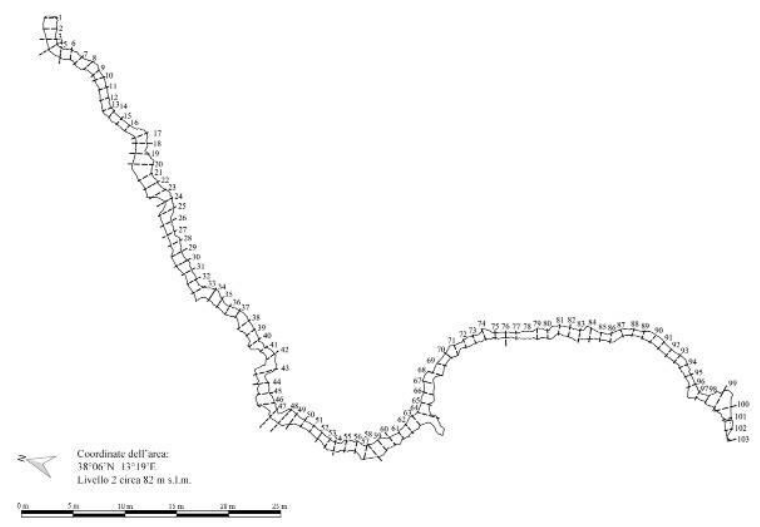

Figure 12. The plan and the cross sections of the qanāt.
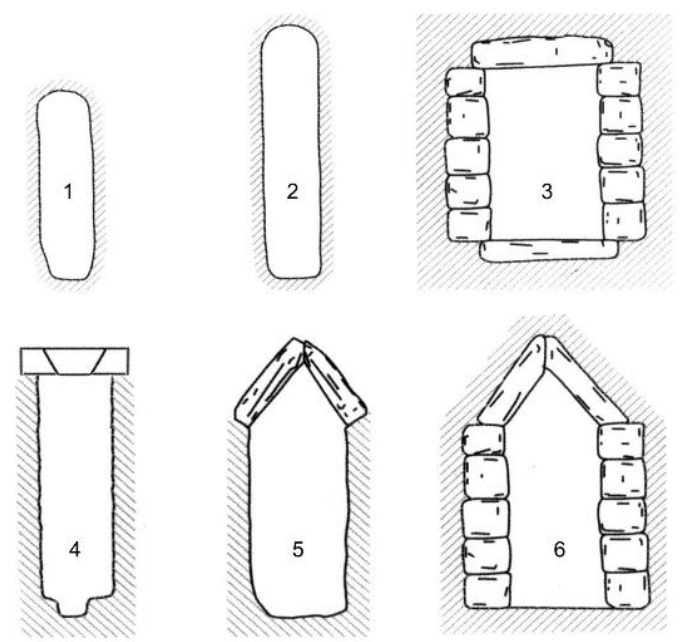

Figure 13. Graphic restitution of the different types of hypogeal sections.

2.2.4 Surface mesh reconstruction: Mesh surface reconstruction from oriented points presents several difficulties that affect the result. The sampling of the points is not uniform. The directions of the normals are not homogeneous due to the imprecision of the sampling. Significant logistical difficulties during survey operations and the presence of undercut and inaccessible areas resulted in some regions of the surface being poor in information.

The procedural steps of post-processing and tessellation of the polygonal mesh were processed within the Leios software (developed by an Italian company, EGS). Using the cloud management and correction tools, overlapping and redundant vertices were detected and eliminated.

Given these unfavourable conditions, different methods with different algorithms (CloudCompare: Poisson Surface Reconstruction and Leios) were employed to reconstruct the surface topology, evaluating the most reliable results in terms of accuracy of the resulting model geometry.

Considering the surface geometry (curvature, contiguity edges and polygonal mesh density) the spatial structure of the model undergoes corrections and some artifacts due to the scanning process are eliminated. The process eliminates recurring topological errors in the geometry.

2.2.5 The platform of virtual reality visualization: The current procedures of study and preservation of $\mathrm{CH}$ have as a scientific reference the acquisition of data and their digitization, aimed at the development of applications for use and knowledge. In this field, the video game industry has contributed in a surprising and attractive way to communicate $\mathrm{CH}$ with visual and interactive tools, involving the public. The so-called "serious games" are an effective tool, especially for the new generations, which elaborates, in a single context, the technological and historical-cultural issues of a topic. Therefore, the video game is one of the valuable resources essential to transmit knowledge and culture. The game environment needs continuous contributions of platform implementation and technological and procedural development. Especially in the case study addressed the design of a graphical and interactive navigation interface, allows to overcome physical, logistical and perceptual barriers, making everyone able to enjoy the underground heritage (Di Paola et al., 2019).

The interface set up, within the environment UnReal Engine (by Epic Games), allows navigation in two interaction modes. 
The first one allows the geo-referencing of the 3D model with infographics and 2D documentation (images, archive photos, diagrams, and drawings), through reference tags. The user can interact with the implemented environmental context with heterogeneous infographics. The second mode allows the user to immerse himself in a realistic scenario and navigate inside the qanāt in game mode, simulating, for example, the light of the protective hat (figs 14-15).

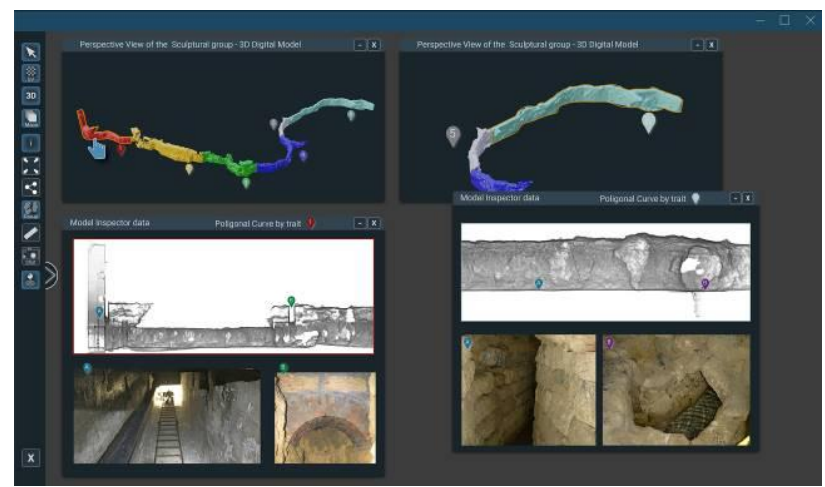

Figure 14. UnReal Engine interface, integrated navigation mode. The geo-referencing of the 3D model with infographics and $2 \mathrm{D}$ documentation, through reference tags.

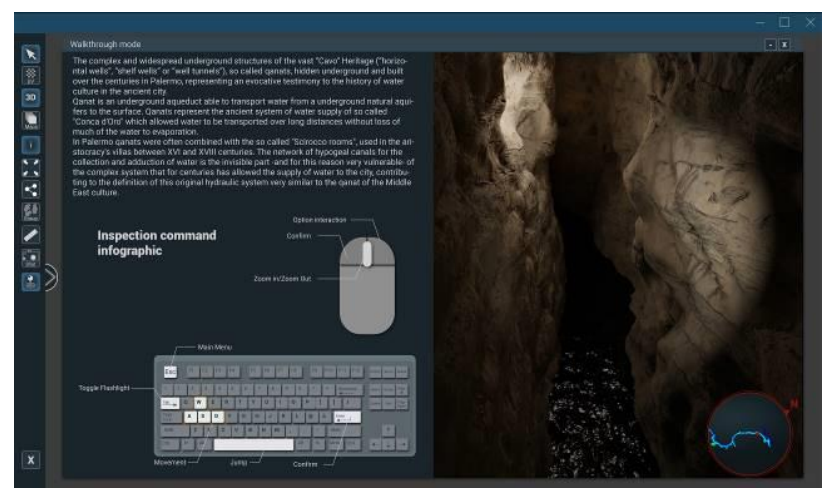

Figure 15. UnReal Engine interface, gaming navigation mode inside the qanāt.

\section{DISCUSSION}

Paradoxically, in urban areas, the discovery of many hypogeal buildings is most often due to localized instability or collapses that appear on the street surface, to fortuitous discoveries following excavations and, only more rarely, to targeted research aimed at verifying references found in archival and bibliographic sources.

The detailed reconstruction of the qanāts network used for water supply not only has a strong historical interest but it is especially important to reconstruct the water flowing conditions also preventing disaster events.

If the typological-functional features of qanāts and serial pits have already been the subject of numerous studies and publications, the constructive aspects and the evaluation of recurrent degradations and instabilities have not been sufficiently investigated. The typological and functional recognition of a historical hypogeal buildings allows to foresee possible risks during exploration, study and eventual fruition, and allows to identify, interpret or predict some recurrent degradation and instability phenomena and possible risks for primary and secondary safety. The assessment of primary safety for hypogeal structures can be evaluated at two different scales: the urban macro-scale, where the presence of the cavity may cause collapses and/or disruption to overlying structures, and the small scale related to safety within the cavity itself.

Contrary to underground caves of considerable size, qanāts are generally characterized by a more linear planimetric pattern and smaller cross sections. With regard to instabilities, these can derive either from intrinsic causes (such as congenital weaknesses in the construction phase) or from indirect causes due to environmental conditions changes (such as water level variations, inflowing water dispersed by aqueducts and sewers) or to anthropic alterations. In the latter case the alteration can be either of direct type on the artefact (robbery quarries, interference with new excavations, deep foundation construction), or because of lack of maintenance (silting up of the tunnels), or of indirect type determined by a variation of the channels shape (variation of loads due to new construction, planting of tall trees with invasive root systems) (Cristiano, 2017; Ferriello, 2006).

The paper contributes to the definition of an innovative methodology, aimed at a multidisciplinary reading for the protection and conservation of this fragile and precious invisible Heritage (fig. 16). By using the collected data the reconstruction of the qanāt "Gesuitico alto" has been operated identifying very narrow cross-sections. It should be noted that the shape of the cross-section could strongly affect the flow velocity and, consequently, the presence of sand/sediments deposited on the bottom. Sediments deposition can determine a progressive reduction of the effective flowing section and the transition to under pressure flow motion. The detailed identification of the longitudinal channel's bottom slope is also important both for identifying flow velocity and for evaluating the potential interactions with monitoring operations.

The georeferencing phase of the acquired data of the hypogeal system with the surface terrain is in progress. The procedure will allow to capture the depth relationship between the underground structures and the ground. Orientation is being recorded using topographic and GPS techniques, taking advantage of the entrances of the two entrance shafts.
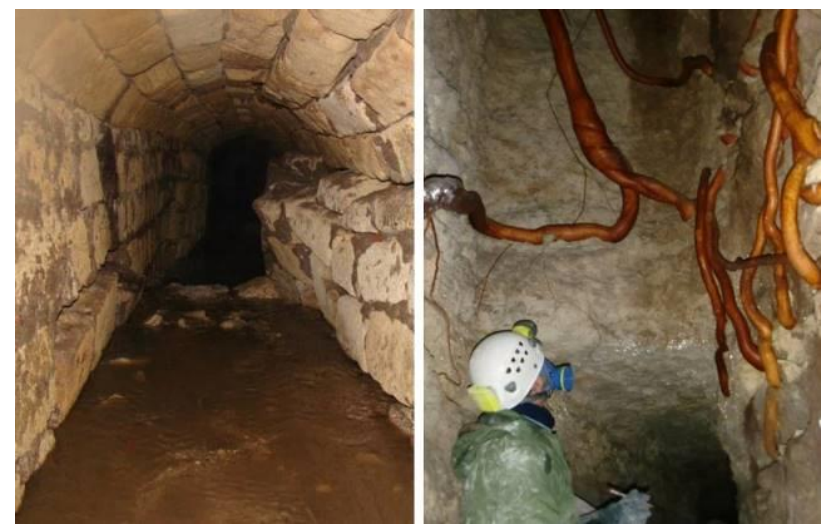

Figure 16. Deterioration due to sidewall collapse and degradation due to root action (Photographic archives CAI Palermo Speleological Group).

\section{CONCLUSIONS}

During the $20^{\text {th }}$ century, the lack of maintenance and the loss of interest in the "invisible" built heritage and the ancient water supply system of Palermo - considered unhygienic - have physically erased, and even from memory, the existence of many hypogeal buildings.

Today a new interest seems to emerge from the studies produced in recent years. These studies direct towards a 
recovery that takes into account, where feasible, the possible rehabilitation of the original function and leads to an enhancement of this heritage as a resource, even in a perspective of sustainable use. Although less easy to visit, the qanāts, especially the "Gesuitico Alto" one, are another attraction for many users who are simply attracted by the fascinating similarity with Middle Eastern culture, and for scholars from different disciplines who see their interests converge around the theme of the relationship between city and water. With respect to this theme, the valorisation is oriented towards the rediscovery and systematization of all the surviving elements, visible and invisible, of the historical "system" of water management in Palermo, that, often, fed the fishponds of many Normans palatial buildings. In this case, the need for conservation is not determined by the "value" of the single artifact, but by a broader vision of the complex relationship between Norman buildings (that already exist), the city and water resources, also in relation to the temporal and cultural transversality that characterizes it. In the field of iHeritage project the investigation of qanāts and hypogeal building heritage of Norman palaces in Palermo will be further implemented and development of measurement and 3D representation and visualization will be carried out with the aim to spread knowledge of Norman epigeal and hypogeal building heritage and allow to many people as possible to visit them with the support of VR and/or AR tools.

\section{ACKNOWLEDGEMENTS}

The Authors thank the Microgeo Srl Company (www.microgeo.it) who provide us the ZEBHorizon system, in particolar thank to Francesco Battimelli e Gianfranco Lupo. The Authors thank, also, the Speleo Team CAI Palermo for its precious assistance in the measurement campaign.

\section{REFERENCES}

De Naeyer, A., Arroyo, S.P., and Blanco, J.R., 2000. Krakow Charter 2000: Principles for Conservation and Restoration of Built Heritage. Available online: http://hdl.handle.net/1854/LU-128776 (accessed on 25 June 2021).

Beraldin, J.A., Picard, M., Bandiera, A., Valzano, V., Negro, N., 2011. Best Practices for the 3D Documentation of the Grotta dei Cervi of Porto Badisco, Italy. Proc. SPIE, Vol. 7864, pp. 78640J-78640J-15.

Bosse, M., Zlot, R., Flick, P. Zebedee, 2012. Design of a springmounted 3-d range sensor with application to mobile mapping. IEEE Trans. Robot, 28, 1104-1119.

Cristiano, M., 2017. La conoscenza del sottosuolo di Napoli per la mitigazione del rischio di crolli e dissesti. In: Proceedings of Colloqui.AT.e. Demolition or reconstruction. Monfalcone: EdicomEdizioni.

Di Filippo, A., Sánchez-Aparicio, L.J., Barba, S., MartínJiménez, J.A., Mora, R., González Aguilera, D., 2018. Use of a Wearable Mobile Laser System in Seamless Indoor 3D Mapping of a Complex Historical Site. Remote Sensing, MDPI, 10 (12), https://doi.org/10.3390/rs10121897.

Di Paola, F., Inzerillo, L., and Alogna, Y., 2019. A gaming Approach for Cultural Heritage knowledge and dissemination, Int. Arch. Photogramm. Remote Sens. Spatial Inf. Sci., XLII-
2/W15, 421-428, https://doi.org/10.5194/isprs-archives-XLII-2W15-421-2019.

Ferriello, G., 2006. L'estrazione delle acque nascoste. Scientific and technical treatise di Karaji. Torino: Kim Williams Books.

Galeazzi F, Moyes, H., Aldenderfer, M., 2014. 3D Documentation in Archaeology: Recording Las Cuevas Site, Chiquibul Reserve, Belize. In: Archaeology in the Digital Era Volume II, Amsterdam University Press, Amsterdam, pp. 350362.

GeoSLAM Technology, ZEB-Horizon Solution Brochure. Available online:

https://geoslam.com/wp-content/uploads/2020/07/ZEB-

Horizon-product-card.pdf (accessed on 25 June 2021).

Kalay, Y.; Kvan, T.; Affleck, J., 2007. New Heritage: New Media and Cultural Heritage, 1st ed.; Routledge: London, UK, ISBN 978-0415773560.

Lagüela, S., Dorado, I., Gesto, M., Arias, P., GonzálezAguilera, D., Lorenzo, H., 2018. Behavior Analysis of Novel Wearable Indoor Mapping System Based on 3D-SLAM. Sensors, 18, 766. https://doi.org/10.3390/s18030766.

Lehtola, V.V., Kaartinen, H., Nüchter, A., Kaijaluoto, R., Kukko, A., Litkey, P.; Honkavaara, E., Rosnell, T., Vaaja, M.T., Virtanen, J.-P., 2017. Comparison of the selected state-ofthe-art $3 \mathrm{~d}$ indoor scanning and point cloud generation methods. Remote Sens. 2017, 9, 796.

Nocerino, E., Menna, F., Remondino, F., Toschi, I., RodríguezGonzálvez, P., 2017. Investigation of indoor and outdoor performance of two portable mobile mapping systems. In Videometrics, Range Imaging, and Applications XIV; International Society for Optics and Photonics: Munich, Germany.

Roncat A., Dublyansky Y., Spotl, C., Dorninger P., 2011. Full3D surveying of caves: A case study of Marchenhohle (Austria). Proc. IAMG2011 Conference, pp. 1393-1403.

Todaro, P., 1988. Il sottosuolo di Palermo, Dario Flaccovio Editore, Palermo.

Vinci, C., 2018. Il patrimonio "cavo". Architetture ipogee e spazio idraulico nella piana di Palermo. In ReUSO 2018. L'intreccio dei saperi per rispettare il passato, interpretare il presente, salvaguardare il futurro, pp. 997-1004. Roma, Gangemi Editore International.

Zlot, R., Bosse, M., Greenop, K., Jarzab, Z., Juckes, E., Robert, J., 2013. Efficiently capturing of large, complex Cultural Heritage sites with a handheld 3D mobile laser mapping system. In: Journal of Cultural Heritage, Vol. 15(6), pp. 670-678.

\section{APPENDIX}

Author Contributions: All authors conceived and designed the experimental campaign; all authors wrote the introduction, the discussion and the conclusions; F.D.P. performed and wrote the pre-processing and post-processing steps.

Funding: This paper was also framed within "iHERITAGE. ITC Mediterranean Platform for UNESCO. Cultural Heritage" project (B_A.2.1_0056), financed by ENI CBC MED Program 2014-2020. 